\title{
Effects of Genetic Captive-Breeding Protocols on Sperm Quality and Fertility in the White-Footed Mouse ${ }^{1}$
}

\author{
Aurelio F. Malo, ${ }^{2,3,4,5}$ Felipe Martinez-Pastor, ${ }^{4,6,7,8}$ Glen Alaks, ${ }^{4}$ Jean Dubach, ${ }^{4}$ and Robert C. Lacy ${ }^{4}$ \\ Center for Conservation and Evolutionary Genetics, ${ }^{3}$ National Zoological Park, Smithsonian Institution, Washington, DC \\ Department of Conservation Science, ${ }^{4}$ Chicago Zoological Society, Brookfield, Illinois \\ Section of Ecology and Evolution, ${ }^{5}$ Silwood Park, Imperial College London, Ascot, Berkshire, United Kingdom \\ ITRA-ULE, INDEGSAL, ${ }^{6}$ and Molecular Biology, ${ }^{7}$ University of León, León, Spain \\ Biology of Reproduction Group, ${ }^{8}$ National Wildlife Research Institute (IREC, UCLM-CSIC-JCCM), Albacete, Spain
}

\section{ABSTRACT}

Mice (Peromyscus leucopus noveboracensis) from a captivebreeding program were used to test the effects of three genetic breeding protocols (minimizing mean kinship [MK], random breeding, and selection for docility [DOC]) and inbreeding levels on sperm traits and fertility. Earlier, in generation 8, one DOC replicate went extinct because of poor reproductive success. By generation 10, spermatozoa from DOC mice had more acrosome and midpiece abnormalities, which were shown to be strong determinants of fertility, as well as lower sperm production and resistance to osmotic stress. In addition, determinants of fertility, including male and female components, were assessed in a comprehensive manner. Results showed that the probability $(P)$ of siring litters is determined by sperm number, sperm viability, and midpiece and acrosome abnormalities; that the $\boldsymbol{P}$ of siring one versus two litters is determined by tail abnormalities; and that the total number of offspring is influenced by female size and proportion of normal sperm, showing the relative importance of different sperm traits on fertility. On average, males with $20 \%$ normal sperm sired one pup per litter, and males with $70 \%$ normal sperm sired eight pups per litter. Interestingly, the proportion of normal sperm was affected by docility but not by relatively low inbreeding. However, inbreeding depression in sperm motility was detected. In the MK group, inbreeding depression not only affected sperm motility but also fertility: An increase in the coefficient of inbreeding ( $f$ ) of 0.03 reduced sperm motility by $30 \%$ and translated into an offspring reduction of three pups in second litters. A genetic load of 48 fecundity equivalents was calculated.

conservation breeding, docility, genetic adaptation to captivity, inbreeding depression, mean kinship, Peromyscus leucopus, reproductive success, testosterone

\footnotetext{
${ }^{1}$ Supported by the IMLS Conservation Project Support Grant IC-03-020186-02. A.F.M. was a recipient of an MEC/Fulbright fellowship from the Spanish Ministry of Education and Science (FU2005-0893) and a Marie Curie fellowship from the European Union (PIEF-GA-2008220322). F.M.-P. was supported by the Juan de la Cierva and Ramón y Cajal programs from the Spanish Ministry of Science and Innovation and by a travel grant from the University of Castilla-La Mancha.

${ }^{2}$ Correspondence: FAX: 44020020759 42339;

e-mail: a.malo@imperial.ac.uk
}

Received: 13 April 2010

First decision: 18 May 2010.

Accepted: 19 May 2010.

(C) 2010 by the Society for the Study of Reproduction, Inc.

elSSN: 1529-7268 http://www.biolreprod.org

ISSN: 0006-3363

\section{INTRODUCTION}

Captive breeding programs have helped species, originally either highly endangered or no longer found in their natural habitats, to be successfully reintroduced into the wild. Such programs have already saved several species from extinction. The examples of the California condor, (Gymnogyps californianus), Guam rail (Gallirallus owstoni), European bison (Bison bonasus), Père David's deer (Elaphurus davidianus), Przewalski's horse (Equus ferus przewalskii), and black-footed ferret (Mustela nigripes) are notable [1]. However, whereas many other species have been reintroduced after captivebreeding programs, the overall success of these reintroductions is still low [2]. The reasons for this low success are multiple. One possible explanation that needs to be tested experimentally is that captive-breeding programs cause genetic changes that negatively impact fitness-related traits after animals have been returned to their natural habitats $[3,4]$.

Captive-breeding programs cause three types of genetic effects: genetic drift, inbreeding, and genetic adaptation to captivity. Genetic drift adds random variation, and it becomes larger as populations decline in size. Inbreeding depression (i.e., the decrease in fitness brought about by inbreeding) is thought to result mainly from the expression of mildly deleterious mutations in homozygotes [5] and is a serious threat to captive and natural populations $[6,7]$ because of its effects on juvenile survival, size, and life span [8-11]. Inbreeding is difficult to avoid in captive-breeding programs due to low numbers of founders, small population sizes, and logistic constraints regarding the translocation of animals for breeding [12].

Genetic adaptation to captivity can be defined as the change in the genetic makeup of a population that occurs when the selection forces change from those imposed by the natural environment to those imposed by the captive environment. Artificial selection for docility is expected to be a common cause of genetic adaptation to captivity in captive-breeding programs, because more docile animals tend to breed more than the average. This happens either because more aggressive or stressed animals are more likely to be injured, die from trauma, or reproduce poorly in the confined spaces of captivity or because animal managers might favor animals that are easier to handle. The efficiency of selection decreases with decreasing population size [13], and because captive populations are generally small, genetic adaptation to captivity may not play a significant role in captive-breeding programs [14]. Currently, however, no estimations are available regarding the strength of artificial selection for docility in captivity. 
Understanding the impact of inbreeding on fecundity is central both to evolutionary biology [15] and to conservation biology [16]. During the past two decades, growing evidence has indicated the effects of inbreeding on male reproductive quality in mammals. Inbred dogs and cats experience reduced reproductive performance, and effects of inbreeding on sperm quality have been shown to be responsible [17-19]. Other studies with inbred or endangered populations of wild carnivores (e.g., lions [Panthera leo; 20], cheetahs [Acinomyx jubatus; 21, 22], and Florida panthers [Puma concolor; 23]) have shown poor male reproductive quality.

Classical studies pointed out the deleterious effects of inbreeding on sperm quality [24]. In studies with captive populations of endangered species, the Sri Lankan leopard (Panthera pardus kotiya) presented a high frequency of sperm abnormalities [25], and the clouded leopard (Neofelis nebulosa) presented severe spermatogenic dysfunction [26]. These captive populations are small, and they have descended from few founders. However, those studies did not report level of inbreeding. Two different studies at the population level, one in natural populations of lions [20] and another in captive populations of three species of gazelles (Gazella dama mhorr, Gazella dorcas neglecta, and Gazella cuvieri) [27-29], found similar results, with a negative correlation between sperm quality and the average inbreeding levels of the population or species. Only one study (in G. cuvieri) has shown a direct negative relationship between coefficient of inbreeding $(f$ range, 0.04-0.24) and sperm quality at the individual level [30]. Another study in captive populations of Mexican wolf (Canis lupus baileyi) [31] found a similar result, although in this case, the comparison involved pooling subjects from different captive populations ( $f$ range, $0-0.6$ ). Regarding sperm production, negative [32] and positive [33] relationships between inbreeding and sperm production have been reported.

These studies suggest that in captive-breeding programs, inbreeding brings about negative effects on sperm quality. However, in most of these studies, the effects of inbreeding were not quantified, and artificial selection for docility or other forms of adaptation to captivity were not considered as possible factors affecting those results. Furthermore, in multigenerational captive-breeding programs, the levels of inbreeding will often be correlated with the levels of selection for docility and unintentional adaptation to captivity. Thus, it remains to be substantiated whether the decline in sperm traits during captive-breeding programs results from inbreeding effects or from artificial selection for docility or other traits favored under captive conditions.

Captive-breeding programs for endangered species typically use a mating protocol that minimizes mean kinship (MK) in the population $[34,35]$. The effectiveness of the MK protocol in terms of maintaining heterozygosity and allelic diversity and minimizing inbreeding has been shown theoretically through simulations [34, 36, 37] and experimentally in Drosophila sp. [38]. However, the effects of following such a protocol to maintain genetic diversity have not been tested empirically on the vertebrate species that conservation breeding programs often target.

In the present study, we used a population of a promiscuous wild species (white-footed mice, Peromyscus leucopus noveboracensis), kept for 10 generations in captivity, to address the genetic effects of three different captive-breeding protocols (minimizing $\mathrm{MK}$, selection for docility [DOC], and random breeding with accumulated inbreeding [RAN]) on sperm traits and fertility. Given that one line in the DOC protocol went extinct in generation 8 , our expectation was that selection for docility might bring about more deleterious effects on fertility than the RAN and MK protocols. Opportunistically, we also tested the effect of inbreeding levels on sperm traits and fertility. Lastly, we tested the effects of sperm traits on fertility.

\section{MATERIALS AND METHODS}

\section{Mice}

In October 2001, fifty-one white-footed mice were trapped at Volo Bog State Natural Area (Lake County, IL, USA). After quarantine and disease testing, mice were brought into a captive environment at the Chicago Brookfield Zoo research facilities to set up the founder population. Sampling theory estimates the amount of heterozygosity that can be retained from a wild to a founder population [39]. On the basis of this theory, it is assumed that no more than 20 individuals are required to establish a captive population [40, $41]$, because they are expected to represent, on average, $1-(1 /(2 \mathrm{~N}))$, or $97.5 \%$, of the wild genetic diversity and, thus, nearly all wild additive genetic variance (for details, see [42]). Nineteen pairings were set up, of which 12 produced litters. The most productive 10 pairs gave birth to more than 240 individuals in 5-10 litters each. These progeny were randomly allocated to six experimental groups, representing two replicates for each of three breeding protocols (20 pairs per protocol and replicate) and subsequently bred in captivity for 10 generations. The three breeding protocols were as follows: 1) MK protocol, which minimized mean kinship (maximizes gene diversity) by pairing the males and the females with the lowest average kinships to the rest of the population; 2) DOC protocol, in which artificial selection for docility was practiced by pairing the males and female with the lowest scores for gnawing and flipping behaviors; and 3) RAN protocol, in which individuals were assigned to pairings in a random manner. The MK protocol followed the genetic management strategy used by many zoos. The DOC protocol aimed to mimic the kinds of purposeful or inadvertent selections for docility that often occur in captive-breeding programs. Two replicate populations were used per protocol. The six populations have never interbred. The data presented in this paper correspond to the 10th generation of captive breeding. The animal care protocols and experiments described here complied with all current laws and were approved by the Animal Care and Use Committee of the Chicago Zoological Society.

\section{Experimental Breeding Protocols}

In the overall experiment, after nine generations in captivity, the accumulation of inbreeding was, on average, higher in DOC mice $(f=0.18)$, lower in RAN mice $(f=0.14)$, and lowest in MK mice $(f=0.12)$. Within protocols, however, differences occurred in the accumulated levels of inbreeding between the two replicates. For the present study, only one replicate per protocol was selected in a design aimed at disentangling the effects of inbreeding and artificial selection for docility. For the MK protocol, the replicate was selected randomly $(\mathrm{MK} 2, f=0.11)$. For the DOC protocol, we could only use one replicate (DOC1, $f=0.13$ ), because the DOC2 population had died out at generation 8 because of poor reproductive success. For the RAN protocol, we selected the replicate that had accumulated more inbreeding (RAN2, $f=0.15$ ) to avoid the DOC group being divergent in both inbreeding and selection for docility, potentially confounding these effects. For simplicity, hereafter the replicate number of the protocol will be omitted: MK represents minimal selection for docility and minimal inbreeding $(f=0.11)$, RAN represents minimal selection for docility and maximum inbreeding $(f=0.15)$, and DOC represents maximal selection for docility and intermediate inbreeding $(f=0.13)$. This design allows us to disentangle, in part, the effects of inbreeding and selection for docility.

\section{Sample Size}

Twenty adult males from each breeding protocol $(n=60)$ were paired with females from the same replicate and protocol for a period of up to 70 days. Males were separated from the females after they had produced two litters or when they had been paired for 70 days. Mean age at death was 204 days (SD, 19.9 days; range, 153-241 days), and mean weight was $25 \mathrm{~g}$ (SD, $3.7 \mathrm{~g}$; range, 19.2-40.5 g). This design results in conservative comparisons of fertility between protocols, because males that are unable to sire litters stay paired for more time than males that sire two litters in a short period. For this reason, MK mice were, on average, 23-24 days younger than RAN and DOC mice when killed (Table 1), because MK mice were faster at siring litters. However, age had no effect on any of the sperm quality traits analyzed $(P>$ $0.05)$.

After being separated from the female, every male was left in an individual cage for at least 7 days before it was killed. This was done to allow 
TABLE 1. Descriptive statistics by protocol (mean \pm SEM), variance explained for each trait by protocol after ANOVAs, $P$ values, and summary of Tukey post-hoc tests and significant pairwise comparisons for significant models.

\begin{tabular}{|c|c|c|c|c|c|c|}
\hline Variables & $M K(n=20)$ & RAN $(n=20)$ & $\mathrm{DOC}(\mathrm{n}=20)$ & $R^{2}(\%)$ & $P$ & Tukey post-hoc $^{b}$ \\
\hline$F$ & $0.11 \pm 0.01$ & $0.15 \pm 0.01$ & $0.13 \pm 0.01$ & 88 & $<0.001$ & $R>D>M$ \\
\hline Age (days) & $188.0 \pm 20.6$ & $212.0 \pm 13.5$ & $211.0 \pm 15.8$ & 37 & $<0.001$ & $R=D>M$ \\
\hline Body length (mm) & $100.0 \pm 3.1$ & $96.5 \pm 3.1$ & $97.4 \pm 3.5$ & 23 & 0.012 & $M>R=D$ \\
\hline Kinship & $0.12 \pm 0.01$ & $0.16 \pm 0.01$ & $0.15 \pm 0.01$ & 89 & $<0.001$ & $\mathrm{R}>\mathrm{D}>\mathrm{M}$ \\
\hline Testes weight (g) & $0.51 \pm 0.14$ & $0.58 \pm 0.16$ & $0.46 \pm 0.09$ & 15 & 0.017 & $M=R>D$ \\
\hline Sperm numbers $\left(\times 10^{6}\right.$ sperm $\left./ \mathrm{ml}\right)$ & $141 \pm 71.1$ & $165 \pm 81.6$ & $101 \pm 25.6$ & 13 & 0.008 & $M=R>D$ \\
\hline Sperm motility $(\%)$ & $77.2 \pm 14.1$ & $71.5 \pm 15.7$ & $72.0 \pm 16.4$ & 8 & 0.237 & - \\
\hline Sperm acrosome integrity (\%) & $84.1 \pm 7.47$ & $84.2 \pm 8.736$ & $85.9 \pm 4.8$ & 2 & 0.697 & - \\
\hline Normal sperm morphology (\%) & $38.9 \pm 18.3$ & $43.9 \pm 20.4$ & $36.1 \pm 18.0$ & 3 & 0.633 & - \\
\hline Head abnormality (\%) & $1.0 \pm 0.33$ & $0.2 \pm 0.08$ & $0.8 \pm 0.36$ & 7 & 0.129 & - \\
\hline Acrosome abnormality (\%) & $0 \pm 0$ & $0 \pm 0$ & $0.2 \pm 0.07$ & 20 & 0.002 & $M=R<D$ \\
\hline Midpiece abnormality (\%) & $3.7 \pm 0.68$ & $2.7 \pm 0.56$ & $5.85 \pm 1.13$ & 11 & 0.031 & $\mathrm{R}<\mathrm{D}$ \\
\hline Bent midpiece $(\%)$ & $5.8 \pm 1.21$ & $6.1 \pm 1.23$ & $6.63 \pm 0.69$ & 0 & 0.858 & - \\
\hline Swollen midpiece (\%) & $45.4 \pm 3.84$ & $44.1 \pm 4.85$ & $42.7 \pm 3.60$ & 0 & 0.894 & - \\
\hline
\end{tabular}

a Values are presented as the mean \pm SEM. Variance is explained for each trait by protocol after ANOVA.

${ }^{b} \mathrm{D}, \mathrm{DOC} ; \mathrm{M}, \mathrm{MK} ; \mathrm{R}, \mathrm{RAN}$.

the replenishment of sperm reserves and to minimize the female presence- and copulation-induced effects on sperm reserves. By doing this, we aimed to further standardize the conditions for males before sperm collection and to minimize residual variance in sperm numbers.

\section{Spermatozoa Collection}

After males were killed, testes were extracted from the abdominal cavity. Testes weight was obtained by adding left and right testis weight after separating the epididymides. The dissected cauda epididymides and vasa deferentia were stored in $0.5 \mathrm{ml}$ of modified Tyrode-Hepes buffer (mT; 300 $\mathrm{mOsm} / \mathrm{kg}, \mathrm{pH}$ 7.4) covered with paraffin oil in a Petri dish at $37^{\circ} \mathrm{C}$. Connective tissue, fat, and blood vessel remains were separated under a binocular microscope. The cauda epididymides and vasa deferentia were then transferred to another Petri dish with $0.5 \mathrm{ml}$ of clean $\mathrm{mT}$. To extract the sperm, the cauda epididymides were repeatedly punctured with a sterile needle for $90 \mathrm{sec}$ and gently handled to help the contents out of the tubules and vasa deferentia. Samples were allowed to rest for $5 \mathrm{~min}$ before removal of the remaining tissue. Then, sperm was transferred to a $1.5-\mathrm{ml}$ Eppendorf tube resting in a warm bath at $37^{\circ} \mathrm{C}$.

\section{Sperm Trait Measures}

After spermatozoa collection, sperm traits were measured (Supplemental Table S1; all Supplemental Data are available online at www.biolreprod.org). Previous research has shown that sperm number [43], motility [44, 45], viability [46, 47], as well as morphology and acrosome integrity (\%NAR) [45, $48,49]$ are associated with fertility. Osmotic resistance has been shown to be affected by the genetic background of mice [50,51]. In addition, sperm motility is substantially more sensitive to osmotic stress compared to the sensitivity of other sperm parameters [52]. Thus, we included a sperm osmotic resistance test [53] to detect changes in membrane resistance caused by altered spermatogenesis or sperm maturation.

Total sperm number. Total sperm number was calculated as the volume of sperm medium $(0.5 \mathrm{ml}$ of $\mathrm{mT}$ media) multiplied by sperm concentration $(\mathrm{sperm} / \mathrm{ml})$ as assessed with a hemocytometer. This gives an estimate of sperm production to supplement measures of testes size.

Sperm motility. Sperm motility was subjectively assessed with a phasecontrast microscope at $37^{\circ} \mathrm{C}$ using a slide warmer. The percentage of motile spermatozoa (0-100\% using increments of 5\%) was recorded.

Sperm viability, morphology, and \%NAR. Sperm viability, morphology, and \%NAR were assessed under a bright field in 200 spermatozoa from smears stained with eosin-nigrosin and mounted in DPX (Fisher Scientific). Regarding sperm morphology, samples were classified according to morphological abnormalities of the head region (acrosome and abnormal heads), midpiece (bent, swollen, or other abnormalities), and tail. The proportion of normal spermatozoa was calculated as the proportion of sperm not presenting abnormalities in any of these components (Supplemental Fig. S1).

Sperm osmotic resistance. Sperm osmotic resistance was quantified as the value for sperm motility reached after an osmotic stress test. In this test, an aliquot of the sperm sample was added to a hyperosmotic medium $(500 \mathrm{mOsm} /$ $\mathrm{kg}$ ) at $37^{\circ} \mathrm{C}$, and $5 \mathrm{~min}$ later, distilled water was added to the sperm sample to restore isosmotic conditions. Sperm motility was reassessed after $5 \mathrm{~min}$.

\section{Genetic Load}

To calculate the average effect of accumulated detrimental genes per individual on fertility fitness, and to put this measure onto the same scale of fitness as that used for effects of inbreeding on survival [54], we calculated fecundity equivalents $[55,56]$. For the present study, the number of fecundity equivalents per diploid genome was calculated as twice the slope $(2 B)$ of the regression of the natural $\log (\ln )$ of relative fecundity $\left(N / N_{\max }\right)$ on the coefficient of inbreeding $(f)$ using the following formula:

$$
\ln \frac{N}{N_{\max }}=-A-B f
$$

where $N$ is fecundity measured as the number of progeny produced, $\mathrm{N}_{\max }$ is the maximum fecundity, $A$ is the intercept at zero inbreeding, and $B$ represents the number of fecundity equivalents per haploid genome. (For more details, see Supplemental Text).

\section{Statistical Analyses}

To test for confounding associations, we first explored whether sperm traits were associated with age or body weight. We found no effects of age on either testes size or the sperm quality traits analyzed $(P>0.2, \mathrm{n}=60)$ except for osmotic resistance, in which a marginally significant effect was observed $(r=$ $-0.25, P=0.05, \mathrm{n}=59)$. As expected, body weight had a significant effect on testes weight $(r=0.42, P<0.001, \mathrm{n}=60)$.

The ANOVA and Levene tests were used to examine for differences between protocols in the mean and variance of the traits analyzed. Multiple ANOVAs reporting Wilks lambda were used to test for multivariate significant differences between protocols in sperm quality traits and, more specifically, sperm morphological abnormalities.

The effect of different sperm traits on fertility was tested while controlling for possible confounding factors, such as allometry, age, inbreeding, and female effects. The effect of the breeding protocol on the probability of siring offspring $(0-1)$ was assessed through chi-square tests. After extinction of one DOC replicate in generation 8, the a priori expectation was that the DOC protocol would have a negative effect in the remaining replicate. To understand the sperm determinants of fertility, we used a three-step approach. Using logistic regression analyses and fitting protocol as a categorical predictor, we first tested which sperm traits 


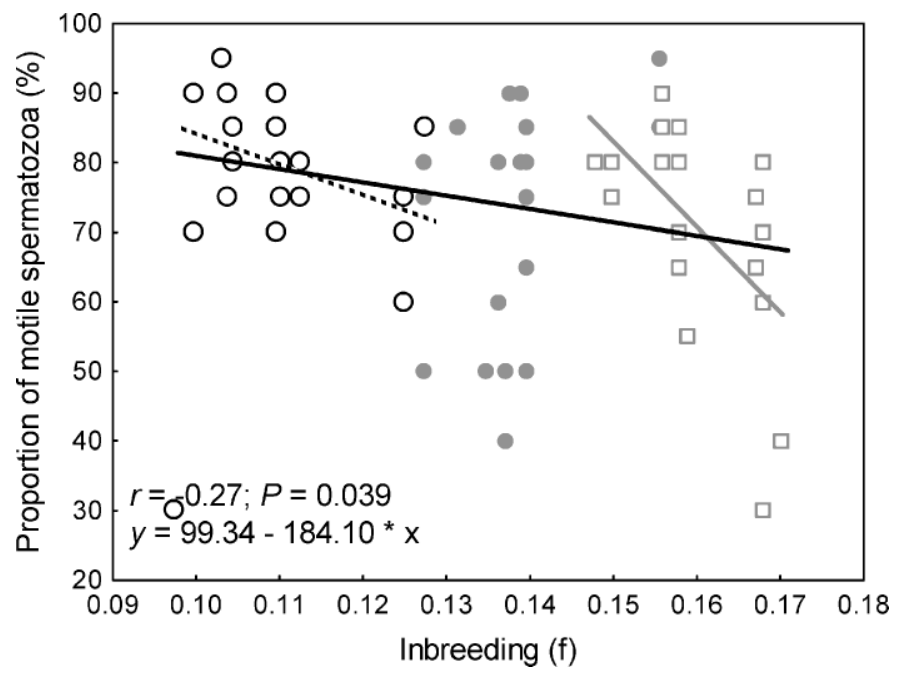

FIG. 1. Relationship between the coefficient of inbreeding $(f)$ and the sperm motility in Peromyscus leucopus $(n=59)$. Overall linear regression (black line) is presented. Gray circles represent DOC mice (linear regression not shown, $P>0.1$ ), empty circles represent $M K$ mice (linear regression: $y=128.45-442.05 x ; r=-0.44, P=0.069)$, and gray squares represent RAN mice (linear regression: $y=266.72-1225.0 x ; r=-0.57, P$ $=0.009)$.

determine siring litter success (0 vs. 1 ). Second, we tested which sperm traits influence the probability of having one versus two litters. Third, we explored which factors determine total number of offspring using generalized linear models. Final models were obtained by stepwise deletion of nonsignificant terms. Given the a priori expectation of the negative relationship between inbreeding levels and sperm traits, we used one-tailed $t$-test to examine for inbreeding depression.

All analyses were conducted in STATISTICA v. 6.0 for Windows [57].

\section{RESULTS}

Effects of Captive-Breeding Protocols and Inbreeding on Testes Size and Sperm Production

Compared with RAN or DOC males, MK males were larger and heavier (Table 1). RAN males had significantly larger testes than DOC males, but not significantly larger than MK males (Table 1). Two generalized linear models including testes size and sperm numbers as the response variables, protocol as a categorical predictor, and body weight and age as continuous predictors rendered two significant maximal models (not shown) that in turn rendered two even more significant minimal adequate models after deletion of the only nonsignificant term: age (testes size: $F_{3.56}=7.15, P<0.001, R^{2}=0.28$; sperm numbers: $F_{3,56}=7.85, P<0.001, R^{2}=0.30$ ). A significant effect of breeding protocol on testes size $\left(F_{256}=\right.$ 3.87, $\left.P=0.026, R^{2}=0.10\right)$ and sperm numbers $\left(F_{2,56}=3.79, P\right.$ $=0.028, R^{2}=0.095$ ) was found, accounting in both cases for the significant effect of body weight on sperm production variables ( $P=0.001$ in both models). The males from the more inbred group (RAN) had significantly larger testes and more sperm than DOC males, but not significantly more than MK males.

We also tested the within-protocol effect of inbreeding levels, conducting regression models including testes size as the response variable and body size and $f$ as the predictors with stepwise backward deletion. In the model conducted for the MK group, but not in the models for the other two groups, inbreeding had a significant positive effect $\left(F_{1,18}=5.57, P=\right.$ $0.029, R^{2}=0.24, \beta \pm \mathrm{SE}=0.49 \pm 0.21[\beta$ represents the slope of the line and defines the sign and size of the effect]) on testes size. This shows that sperm production is positively associated with inbreeding both between and within groups.

\section{Effect of Captive-Breeding Protocols and Inbreeding on Sperm Quality and Fertility}

An overall main multivariate effect of protocol on sperm quality was found (Wilks lambda $=0.65, F_{10,106}=2.51, P=$ $0.009)$. MK and RAN mice presented higher sperm osmotic resistance than DOC mice $\left(F_{2,57}=7.69, P=0.001\right)$. No univariate effects were detected on the other sperm quality traits considered. However, the overall effect of breeding protocol on sperm morphology was also significant (Wilks lambda $\left.=0.59, F_{12,104}=2.61, P=0.004\right)$. More specifically, MK and RAN mice had fewer acrosome and midpiece abnormalities than DOC mice $\left(F_{2,57}=6.91, P=0.002\right.$ and $F_{2,57}=3.68, P=0.031$, respectively) (Table 1$)$.

Regarding inbreeding, a significant negative relationship between $f$ and osmotic resistance was found within the RAN protocol $(r=-0.59, P=0.006, y=215.9-1103 x)$, which was the protocol with the highest levels of inbreeding ( $f$ range, $0.145-0.17)$, but not in MK or DOC protocol ( $f$ range, $0.095-$ 0.125 and $0.125-0.155$, respectively). After pooling males from the three protocols, we tested for an effect of inbreeding by conducting simple regressions for the remaining sperm quality traits. After the removal of a strongly influential outlier (Cook distance, 0.41), a negative relationship was found between $f$ and sperm motility $\left(R^{2}=0.07, P=0.039\right)$ (Fig. 1). This relationship arises from an increased number of animals with low sperm motility at higher levels of inbreeding. The negative effect of $f$ on motility was observable within the two protocols with the highest variation in $f$ (RAN and MK), but not in the DOC protocol, which had little variation in $f$ (Fig. 1).
A
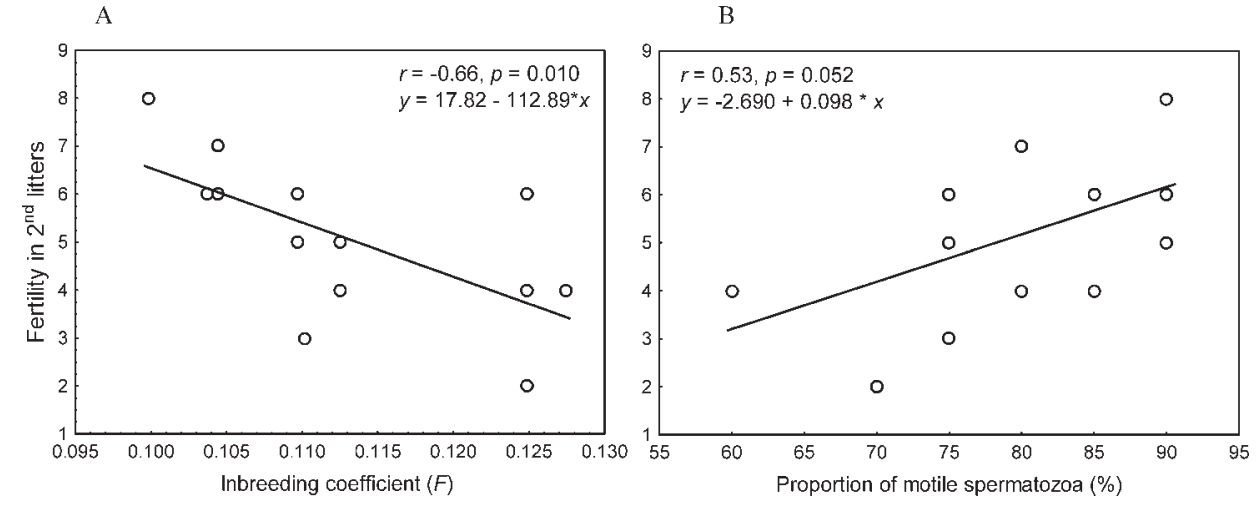

FIG. 2. Linear regressions of fertility on inbreeding levels (A) of the sire and sperm motility $(\mathbf{B})$ in second litters of the MK group. Only individuals that sired at least one offspring are considered. For both graphs, $n=14$; note one and two overlapping data points on $\mathbf{A}$ and $\mathbf{B}$, respectively. 


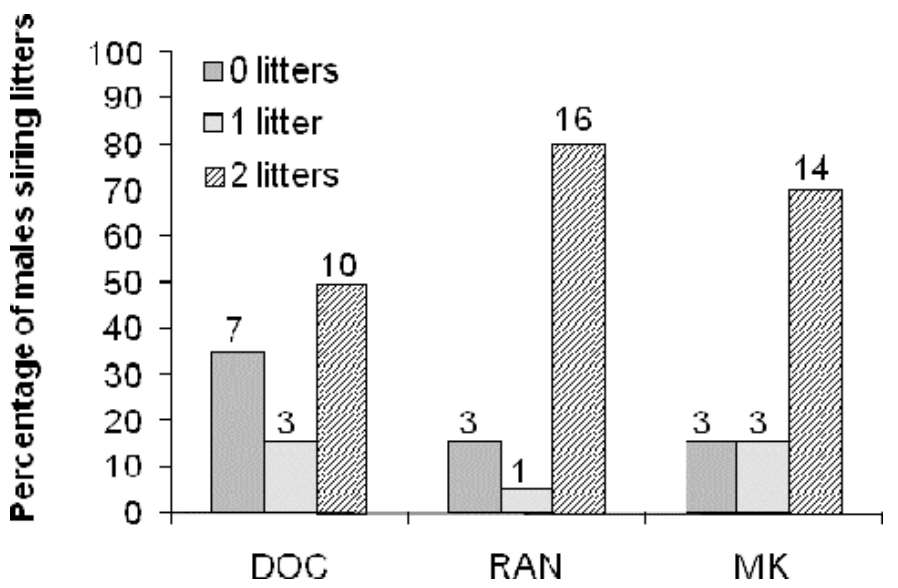

FIG. 3. Differences between breeding protocols in the proportion of males siring zero, one, or two litters after 70 days paired with a female. The number above each bar indicates the number of males within that group $(n=60)$.

We then tested whether this inbreeding depression in terms of sperm motility translated into reductions in total number of offspring or in first or second litters of RAN or MK protocols. We found an effect of inbreeding on fertility in second litters for the MK protocol, apparently driven by the impact of inbreeding on sperm motility (Fig. 2). The same effect was not observed in the first litters, perhaps because of the lower variance $\left(F_{69,80}=1.95, P<0.01\right)$ in and mean $\left(t_{85}=4.42, P<\right.$ 0.001 ) number of pups born in first litters (first litters, $4.1 \pm$ 1.3 ; second litters, $5.3 \pm 1.7$ ). The genetic load was 48.6 fecundity equivalents, as calculated from twice the slope of the regression of fecundity on $f$ for the sire $\left(f_{\text {sire }} ; r=-0.63, P=\right.$ $0.017, y=4.311-24.295 x)$. No effect of $f$ for the dam $\left(f_{\mathrm{dam}}\right)$ on fecundity was detected $(P>0.1)$, although the trend was also negative $(r=-0.45)$.

Given the extinction of one of the DOC replicates in generation 8, we tested whether DOC males, after pairing, failed to produce offspring more frequently than RAN or MK. This was, in fact, the case. RAN and MK pairings failed to produce offspring less than half the number of times that DOC pairings did $\left(12 \%\right.$ vs. $\left.35 \%, \chi_{1}^{2}=4.01, P=0.045\right)$. No differences were found between $\mathrm{MK}$ and RAN mice or between first and second litters $(P>0.05)$. Figure 3 shows a clear trend toward RAN and MK being more productive than DOC, which had a lower proportion of males siring two litters and a higher proportion of males siring zero litters.

\section{Determinants of Male Fertility}

Effect of sperm traits on probability of siring litters. Only sperm viability and proportion of normal sperm were found to determine the likelihood of siring offspring. Males siring offspring had significantly higher sperm viability (63\% vs. $54 \%, F_{1,57}=5.51, P=0.02$ ) and percentage normal sperm (43\% vs. $27 \%, F_{1,57}=7.02, P=0.01$ ) than those not siring litters (Fig. 4A). A close inspection showed that the sperm normality variables responsible for this effect were midpiece and acrosome abnormalities (Table 2); note that DOC mice had significantly lower values for three of these variables (midpiece abnormalities, acrosome abnormalities, and sperm numbers). We then compared these sperm traits for males siring zero versus males siring two litters. The differences increased even more (results not shown), and another sperm morphology variable (swollen midpiece abnormality) also showed significant differences $(P<0.001)$ between males that sired either zero or two litters. Males that sired zero litters had a mean of $25 \%$ normal sperm, whereas those that sired one or two litters had a mean of $45 \%$ normal sperm $\left(F_{2,57}=3.76, P=0.029\right)$ (Fig. 4C).

Effect of sperm traits on probability of siring one or two litters. After including all sperm traits in the model and DOC, $\mathrm{MK}$, and RAN protocols, the logistic model showed a significant effect on the likelihood of siring one or two litters only for the proportion of tail abnormalities (log-likelihood $=$ $\left.-18.47, \chi^{2}{ }_{1}=4.87, P=0.027\right)$. Males siring one litter had $9 \%$
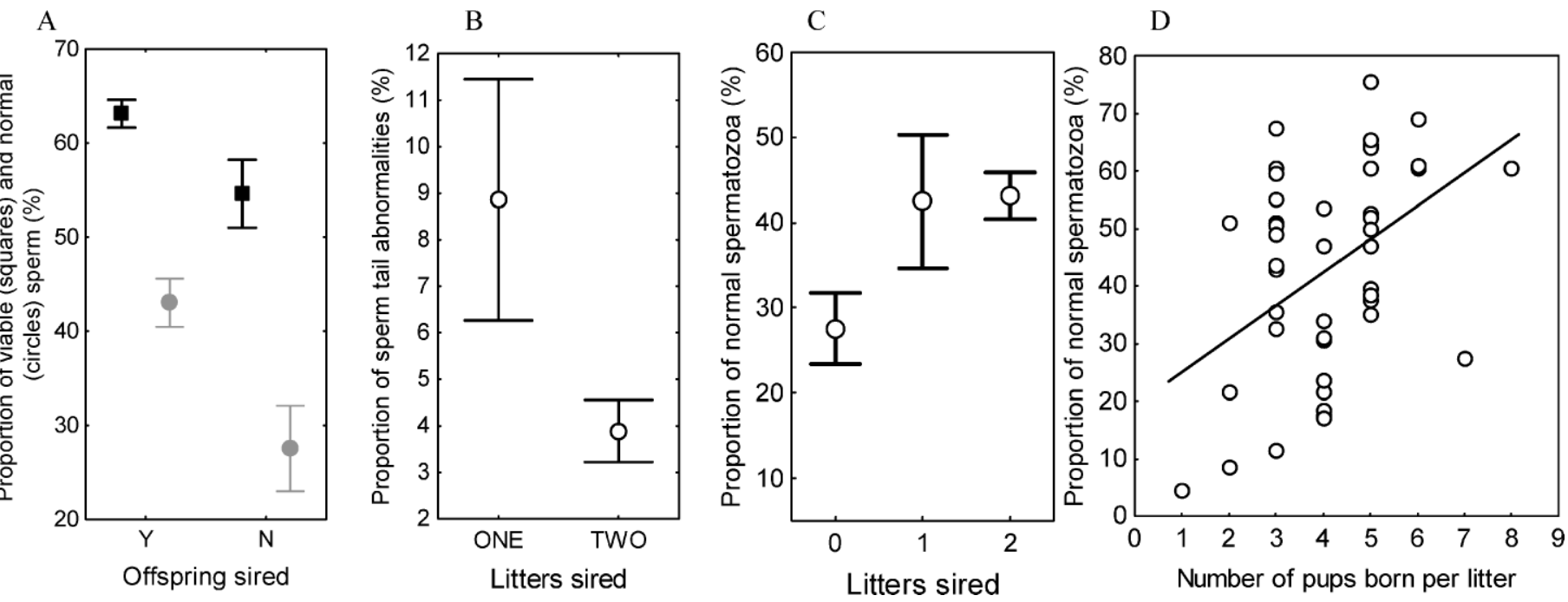

FIG. 4. A) Mean differences in the proportion of viable and normal sperm $\left(F_{1.57}=5.51, P=0.022\right.$ and $F_{1.57}=7.02, P=0.01$, respectively) between males that were able $(\mathrm{Y})$ or unable $(\mathrm{N})$ to sire offspring $(\mathrm{n}=58)$. B) Mean difference in the proportion of tail abnormalities $\left(\mathrm{F}_{1,45}=6.12, P=0.017\right)$ between males that sired one or two litters $(n=46)$. C and $\mathbf{D})$ Relationship between mean percentage normal spermatozoa and number of litters $(\mathbf{C})$ and between mean percentage normal spermatozoa and number of pups born per first litter obtained $(\mathbf{D} ; n=60)$. Axes are reversed for illustration purposes. Linear-regression equation in $\mathbf{D}$ shows regression of number of offspring on percentage normal sperm $(y=2.7501+0.0315 x)$. All analyses were conducted in males after 70 days paired with a female. Error bars indicate 95\% SEM. 
TABLE 2. Logistic regression models (binomial distribution and logit link function) showing the effects of sperm traits that significantly influenced the probability of siring offspring fitted independently in different models. ${ }^{a}$

\begin{tabular}{lccrc}
\hline Parameter & $d f$ & Log-likelihood & \multicolumn{1}{c}{$\chi^{2}$} & $P$ \\
\hline Sperm numbers & 1 & -30.6058 & 7.212 & 0.00724 \\
Sperm viability (\%) & 1 & -33.2089 & 12.418 & 0.00043 \\
Midpiece abnormality & 1 & -32.0917 & 10.183 & 0.00142 \\
Acrosome abnormality & 1 & -29.1142 & 4.228 & 0.03975
\end{tabular}

$\bar{a}$ Best model including the four predictors (Akaike Information Criterion $=$ 49.95; log-likelihood ratio $=19.65 ; P=0.0006$ )

$\pm 2.7 \%$ sperm with abnormal tails as compared to $4 \% \pm 0.7 \%$ in males that sired two litters (Fig. 4B; mean $\pm \mathrm{SE}$ ).

Effect of sperm traits on number of offspring. Failures to breed could have been the result of behavioral or other factors, whereas among pairs that did breed, number of offspring produced might reflect greater effects of sperm quality. Excluding those males with no offspring, an ANOVA showed no significant differences $\left(F_{2,37}=0.479, P=0.62\right)$ between protocols in the total number of offspring. Hence, males from the three protocols were pooled to conduct a multipleregression model including as predictors sperm numbers, five sperm quality traits (\% motile, $\%$ viable, $\%$ normal spermatozoa, \%NAR, and osmotic resistance), dam body length, age, and both $f_{\text {sire }}$ and $f_{\text {dam }}$. The reduced model $\left(R^{2}=0.56, F_{3,35}=\right.$ $15.43, P<0.001)$ only retained three significant variables and showed that dam size was the most important determinant of total number of offspring $\left(R^{2}=0.30, P<0.001\right)$, followed by percentage normal spermatozoa $\left(R^{2}=0.22, P<0.002\right)$ and, lastly, by $\%$ NAR $\left(R^{2}=0.05, P<0.049\right)$. A male having $20 \%$ normal sperm would, on average, sire one pup per litter, and a male having around $70 \%$ normal sperm would, on average, sire eight pups (Fig. 4D). Another model breaking down sperm morphology into its basic components showed that the morphology variable swollen midpiece entirely accounted for the effect on percentage normal spermatozoa, whereas the other components had nonsignificant negative effects. No effect of sperm numbers or testes size was detected, suggesting that sperm quality can be a more important determinant of fertility than sperm production. A generalized linear model including breeding protocol as a covariate rendered identical results. Two more models were conducted for first and second litters separately and produced similar results (models not shown).

\section{DISCUSSION}

To our knowledge, the present study is the first time that the accumulated effects of artificial selection for an aspect of domestication (docility) and inbreeding have been considered jointly. Importantly, in the overall breeding study (Lacy et al., unpublished data), one of the two replicates selected for docility had gone extinct in generation 8 because of increasingly poor reproductive success. To determine if genetic effects negatively affecting sperm traits were responsible for this extinction, we explored the sperm determinants of fertility, and we compared fertility and sperm traits between the remaining docility replicate and the two other breeding protocols. Genetic drift among and between replicates might have reduced our ability to document the effects of inbreeding or selection; however, we were able to document important differential effects in the breeding protocols. The DOC males performed worse than both RAN males (with the highest accumulated inbreeding levels) and MK males, suggesting that selection for docility has more deleterious effects than inbreeding at the $f$ levels considered. This may result from the fact that selection for docility would be expected to have cumulative effects on fitness if an association between the two exists, whereas inbreeding would be expected to show lower association with fitness if inbreeding accumulation is slow enough for purging to operate.

Artificial selection for docility negatively impacted two sperm morphology traits, acrosome and midpiece abnormalities, which together with sperm viability determine the probability of siring litters. This shows that selection for docility can impact fertility through an effect on the proportion of normal sperm, and it suggests a likely pathway through which the docility replicate went extinct in generation 8 . We also show that the proportion of tail abnormalities predicts a second component of fertility, the probability of siring a second litter, and that a third component, total number of offspring, is predicted by the proportion of normal sperm. This hierarchical analysis showing specific contributions of sperm quality parameters to different fertility components strongly emphasizes the multivariate nature of male fertility, explaining, in part, why the sperm determinants of fertility have remained so elusive [58]. Interestingly, we found that dam body size explained a considerable amount of the variance in number of offspring, and including this factor in the model improved our ability to quantify male effects more precisely. In agreement with previous research [45], proportion of normal sperm was the main sperm trait influencing offspring number, stressing the generality of this pattern for mammals. The joint inclusion of sperm production and sperm quality traits in the models allowed us to quantify the effect of each sperm trait independently. Under the assumption that sperm numbers reflect the amount of sperm inseminated, our results suggest that quality is more important than quantity in noncompetitive scenarios.

On top of the increased acrosome and midpiece abnormalities, DOC males also had lower sperm production than RAN males, suggesting that artificial selection for docility can have a stronger negative impact than inbreeding on testes size and sperm production. One possibility is that selection for docility indirectly selects for low testosterone levels [59] and, hence, for smaller testes size [60]. Other effects in the DOC protocol further suggest this possibility. Indeed, the decreased resistance to osmotic stress detected in DOC males could be the result of alterations in spermatogenesis or sperm maturation in the epididymides that reduce the ability of the sperm cell to regulate volume changes [61]. Relatively high androgen levels of dihydrotestosterone (synthesized from testosterone) are known to play a major role in sperm maturation [62-64], which in turn influences osmotic resistance [65]. So, reduced dihydrotestosterone at the site of sperm maturation could have altered osmotic resistance in the DOC males and, potentially, have an impact on fertility [66]. Our undetected effect of protocol on sperm viability and detected effect on sperm morphology also fit this explanation, because spermatozoa remain viable in a low androgen environment [62] whereas sperm morphology parameters tend to be impacted by low androgen levels [63].

Could behavioral selection for docility, however, have indirectly selected for lower testosterone levels? Several lines of evidence suggest this could have been the case [67]. First, testosterone is known to affect body size [68, 69] and testes size [60], and DOC mice suffered a reduction in both body and testes size. Second, testosterone is positively associated with mobility [70] and physical activity [71], which are proxies for the flipping behavior selected against in the DOC protocol. This testosterone-driven effect would also explain the lack of 
differences between RAN and MK males, none of which are expected to suffer a reduction in testosterone levels.

Selection for docility has negative effects on sperm quality traits that impact the probability of siring litters. This should cause us to avoid in conservation breeding programs not only intentional selection for behavioral traits that we think will improve performance in captivity but also unintentional favoring of more docile animals.

Insights regarding the effects of inbreeding were gained by comparing sperm and fertility traits between protocols with different accumulated inbreeding levels and by analyzing the effect of increasing $f$ levels on sperm parameters and fertility within groups. Although the DOC protocol did perform more poorly than the other two protocols, no differences were found in the average levels of sperm quality or fertility reached by MK and RAN males.

The negative effect of inbreeding on sperm motility suggests that nuclear genes are predominantly determining this sperm trait, because we would not expect to detect inbreeding depression on traits encoded by the mitochondrial genome, which is haploid and, therefore, unaffected by inbreeding. This agrees with previous research showing nuclear encoded structures as the main determinants of motility [72]. However, the negative effect of inbreeding was driven by an increased variation in motility with increasing inbreeding, showing that males with higher levels of inbreeding have an increased probability of presenting low sperm motility. Within protocols, this pattern was also followed by RAN and MK mice. This decrease in sperm motility translated into decreased fertility in MK second litters. Surprisingly a 0.03 change in $f$ translated into a $30 \%$ reduction in sperm motility (from $90 \%$ to $60 \%$ ) and resulted in a second-litter size difference of three offspring. The reported genetic load of 48 fecundity equivalents for $\mathrm{MK}$ suggests a high number of genes affecting sperm quality, with even relatively small differences in $f$ (and, thus, differences at a small percentage of loci) resulting in insignificant effects. Again, this would not be a surprise considering that fertility is a multidimensional trait determined by multiple sperm variables, all of which are targets of mutation. It also suggests a larger number of deleterious recessive alleles. Our ability to detect a significant effect of inbreeding on fecundity in the MK protocol, but not in the DOC and RAN protocols, may have been the result of deleterious alleles not yet having been purged from the MK lines (i.e., the more outbred populations with high levels of heterozygosity as opposed to the populations in the other protocols).

Because we found a similar but not significant trend in first litters, one possible explanation is that inbreeding depression was not detected because of a lack of statistical power. Another, more plausible explanation, however, is that inbreeding load increases with age [73], enhancing the chances of detecting inbreeding depression in second litters. This agrees with the age-specific inbreeding depression in terms of male mating success previously shown for Drosophila sp. [74]. The lack of an effect of $f_{\text {dam }}$ on fecundity suggests a lower genetic load for fecundity in females than in males, and it supports previous research in a butterfly that also showed male-biased inbreeding depression [55]. As suggested by those authors, this pattern could be explained by the fact that fertility selection per sperm is expected to be weaker than per egg.

The fact that we have uncovered, at the low levels of $f$ considered in the present study, inbreeding depression only in terms of sperm motility is interesting. Sperm motility is expected to be affected by sperm and seminal traits more than the other variables considered here and, hence, to be potentially determined by a higher number of genes (from sperm morphology, biochemistry, or energetics to seminal parameters). In light of these results, we propose that sperm motility suffers higher mutational load than other sperm traits. This should be expected in that if a low inbreeding level only slightly affects individual sperm traits, the possibility of detecting that effect would less than the possibility for a variable that is cumulatively affected by multiple such traits.

Lastly, the positive relationship between inbreeding and sperm production found between protocols (i.e., the RAN protocol presenting high sperm production) and within protocols could be the result of a compensatory effect between sperm quality and production, as already proposed to explain increased sperm output in individuals with increased abnormal sperm $[33,75]$. Previous research in oldfield mice (Peromyscus polionotus) showed the same pattern, with a transient increase in testes size for low levels of $f$ that is eventually reversed at the higher inbreeding levels [32]. However, we failed to detect a negative correlation between proportion of normal sperm and absolute/relative testes size (results not shown) at the $f$ levels considered, so this question deserves further attention.

Although not as deleterious for reproductive fitness as docility selection, inbreeding also has a negative effect. Sperm quality and fertility decreased with increasing inbreeding in the most outbred protocol after just 10 generations of a captivebreeding program. Several reasons could explain why the effects reported here for low inbreeding levels likely are an underestimate of the negative effects of inbreeding on the main determinants of fertility. First, captivity masks inbreeding effects on male mating success [76], probably because of the absence of male-male competition for mating, which magnifies inbreeding depression [77]. In the present study, males were given 70 days to mate with the female. Second, in captivebreeding programs of vulnerable or endangered species, the founders might already be inbred at a level higher than that tested in the present study and, thus, be vulnerable to more detrimental effects. Lastly, random breeding likely is not being achieved, because logistic constraints in translocating breeding individuals among zoos can generate higher inbreeding levels than expected with more complete mixing.

Overall, our results have shown that 1) different fertility components are affected by different sperm traits; 2) the DOC protocol has the highest negative impact on fertility, and the driver for the extinction of one line of this protocol in generation 8 is likely to have been decreased sperm quality; 3 ) the effect of the overall negative impact of the DOC protocol on fertility is mainly mediated by sperm morphology traits impacting the probability of siring litters; 4) use of the RAN protocol versus use of the MK protocol makes no difference in the average reproductive fitness after 10 generations; 5) negative effects of inbreeding are found within protocols; and 6 ) inbreeding translates into decreased fecundity, at least within the MK protocol, by reducing the size of second litters.

\section{ACKNOWLEDGMENTS}

We thank Jon Ballou, Guy Vanover, and Rachel Kolb for constant support. We also thank Jon Ballou and JoGayle Howard for paperwork that was instrumental in allowing this collaboration to succeed.

\section{REFERENCES}

1. Frankham R, Ballou JD, Briscoe DA. Introduction to Conservation Genetics. Cambridge, UK: Cambridge University Press; 2002.

2. Balmford A. Priorities for captive breeding-which mammals should board the ark? In: Entwistle A, Dunstone $\mathrm{N}$ (eds.), Priorities for Conservation of Mammalian Diversity. Cambridge, UK: Cambridge University Press; 2000:291-307.

3. Lacy RC. Impacts of inbreeding in natural and captive populations of 
vertebrates: implications for conservation. Pers Biol Med 1993; 36:480496.

4. Mace GM, Baillie JEM, Beissinger SR, Redfort KH. Assessment and management of species at risk. In: Soulé ME, Orians GH (eds.), Conservation Biology: Research Priorities for the Next Decade. Washington, DC: Island Press; 2001:11-29.

5. Charlesworth D, Charlesworth B. Inbreeding depression and its evolutionary consequences. Annu Rev Ecol Syst 1987; 18:237-268.

6. Keller LF, Waller DM. Inbreeding effects in wild populations. Trends Ecol Evol 2002; 17:230-241.

7. Leberg PL, Firmin BD. Role of inbreeding depression and purging in captive breeding and restoration programs. Mol Ecol 2008; 17:334-343.

8. Ballou JD. Ancestral inbreeding only minimally affects inbreeding depression in mammalian populations. J Hered 1997; 88:169-178.

9. Lacy RC, Petric AM, Warneke M. Inbreeding and outbreeding depression in captive populations of wild species. In: Thornhill NW (ed.), The Natural History of Inbreeding and Outbreeding. Chicago: University of Chicago Press; 1993:352-374.

10. Ralls K, Ballou JD, Templeton A. Estimates of lethal equivalents and the cost of inbreeding in mammals. Conserv Biol 1988; 2:185-193.

11. Ralls K, Brugger K, Ballou JD. Inbreeding and juvenile mortality in small populations of ungulates. Science 1979; 206:1101-1103.

12. Mace GM, Ballou JD. Population management for conservation. Trends Ecol Evol 1990; 5:102-104.

13. Li W.-H. Maintenance of genetic variability under the joint effect of mutation, selection and random drift. Genetics 1978; 90:349-382.

14. Frankham R. Genetic adaptation to captivity in species conservation programs. Mol Ecol 2008; 17:325-333.

15. Roff DA, DeRose MA. The evolution of trade-offs: effects of inbreeding on fecundity relationships in the cricket Gryllus firmus. Evolution 2001; $55: 111-121$.

16. Ryan KK, Lacy RC, Margulis SW. Impacts of inbreeding on components of reproductive success. In: Holt WV, Pickard AR, Rodger JC, Wildt DE (eds.), Reproductive Science and Integrated Conservation. Cambridge, UK: Cambridge University Press; 2003:82-96.

17. Wildt DE, Baas EJ, Chakraborty PK, Wolfle TL, Stewart AP. Influence of inbreeding on reproductive performance, ejaculate quality and testicular volume in the dog. Theriogenology 1982; 17:445-452.

18. Pukazhenthi BS, Wildt DE, Howard JG. The phenomenon and significance of teratospermia in felids. J Reprod Fertil Suppl 2001; 57: 423-433.

19. Pukazhenthi BS, Neubauer K, Jewgenow K, Howard JG, Wildt DE. The impact and potential etiology of teratospermia in the domestic cat and its wild relatives. Theriogenology 2006; 66:112-121.

20. Wildt DE, Bush M, Goodrowe KL, Packer C, Pusey AE, Brown JL, Joslin $\mathrm{P}$, O'Brien SJ. Reproductive and genetic consequences of founding isolated lion populations. Nature 1987; 329:328-331.

21. O'Brien SJ, Wildt DE, Bush M, Caro TM, FitzGibbon C, Aggundey I, Leakey RE. East African cheetahs: evidence for two population bottlenecks? Proc Natl Acad Sci U S A 1987; 84:508-511.

22. O'Brien SJ, Wildt DE, Goldman D, Merril CR, Bush M. The cheetah is depauperate in genetic variation. Science 1983; 221:459-462.

23. Roelke ME, Martenson JS, O'Brien SJ. The consequences of demographic reduction and genetic depletion in the endangered Florida panther. Curr Biol 1993; 3:340-350.

24. Rice VA, Andrews FN, Warwick EJ, Legates JE. Breeding and Improvement of Farm Animals. In: New York: McGraw-Hill; 1967: 188-190.

25. Brown JL, Wildt DE, Phillips LG, Seidensticker J, Fernando SBU, Miththapala S, Goodrowe KL. Adrenal-pituitary-gonadal relationships an ejaculate characteristics in captive leopards (Panthera pardus kotiya) isolated on the island of Sri Lanka. J Reprod Fertil 1989; 85:605-613.

26. Wildt DE, Howard JG, Hall LL, Bush M. Reproductive physiology of the clouded leopard: I. Electroejaculates contain high proportions of pleiomorphic spermatozoa throughout the year. Biol Reprod 1986; 34: 937-947.

27. Cassinello J, Abaigar T, Gomendio M, Roldan ERS. Characteristics of the semen of three endangered species of gazelles (Gazella dama mhorr, G. dorcas neglecta, and G. cuvieri). J Reprod Fertil 1998; 113:35-45.

28. Garde JJ, Soler AJ, Cassinello J, Crespo C, Malo AF, Espeso G, Gomendio A, Roldan ERS. Sperm cryopreservation in three species of endangered gazelles (Gazella cuvieri, G. dama mhorr, and G. dorcas neglecta). Biol Reprod 2003; 69:602-611.

29. Gomendio M, Cassinello J, Roldan ERS. A comparative study of ejaculate traits in three endangered ungulates with different levels of inbreeding: fluctuating asymmetry as an indicator of reproductive and genetic stress. Proc R Soc Lond B Biol Sci 2000; 267:875-882.
30. Roldan ERS, Cassinello J, Abaigar T, Gomendio M. Inbreeding, fluctuating asymmetry, and ejaculate quality in an endangered ungulate. Proc R Soc Lond B Biol Sci 1998; 265:243-248.

31. Asa C, Miller P, Agnew M, Rebolledo JAR, Lindsey SL, Callahan M, Bauman K. Relationship of inbreeding with sperm quality and reproductive success in Mexican gray wolves. Anim Cons 2007; 10: 326-331.

32. Margulis SW, Walsh A. The effects of inbreeding on testicular sperm concentration in Peromyscus polionotus. Reprod Fertil Dev 2002; 14:6367.

33. Pukazhenthi BS, Neubauer K, Jewgenow K, Howard J, Wildt DE. The impact and potential etiology of teratospermia in the domestic cat and its wild relatives. Theriogenology 2006; 66:112-121.

34. Ballou JD, Lacy RC. Identifying genetically important individuals for management of genetic variation in pedigreed populations. In: Ballou JD, Gilpin M, Foose TJ (eds.), Population Management for Survival and Recovery: Analytical Methods and Strategies in Small Population Conservation. New York: Columbia University Press; 1995:76-111.

35. Lacy RC. Clarification of genetic terms and their use in the management of captive populations. Zoo Biol 1995; 14:565-577.

36. Fernandez J, Toro MA. The use of mathematical programming to control inbreeding in selection schemes. J Anim Breed Genet 1999; 116:447-466.

37. Toro MA, Silio L, Rodriganez J, Fernandez J. Optimal use of genetic markers in conservation programs. Genet Sel Evol 1999; 31:255-261.

38. Montgomery ME, Ballou JD, Nurthen RK, England PR, Briscoe DA, Frankham R. Minimizing kinship in captive breeding programs. Zoo Biol 1997; 16:377-389.

39. Crow JF, Kimura M. An Introduction to Population Genetics Theory. New York: Harper and Row; 1970.

40. Foose TJ. The relevance of captive populations to the conservation of biotic diversity. In: Schonewald-Cox CM, Chambers SM, MacBryde B, Thomas WL (eds.), Genetics and Conservation: A Reference for Managing Wild Animal and Plant Populations. Menlo Park, CA: Benjamin/Cummings; 1983:374-401.

41. Soulé ME, Gilpin M, Conway W, Foose T. The Millennium Ark: how long a voyage, how many staterooms, how many passengers? Zoo Biol 1986; 5:101-113.

42. Lacy RC. Managing genetic diversity in captive populations of animals. In: Bowles ML, Whelan CJ (eds.), Restoration of Endangered Species. Cambridge, UK: Cambridge University Press; 1994:63-89.

43. Dewsbury DA, Sawrey DK. Male capacity as related to sperm production, pregnancy initiation, and sperm competition in deer mice (Peromyscus maniculatus). Behav Ecol Sociobiol 1984; 16:37-47.

44. Jasko DJ, Little TV, Lein DH, Foote RH. Comparison of spermatozoal movement and semen characteristics with fertility in stallions: 64 cases (1987-1988). J Am Vet Med Assoc 1992; 200:979-985.

45. Malo AF, Garde JJ, Soler AJ, García AJ, Gomendio M, Roldan ERS. Male fertility in natural populations of red deer is determined by sperm velocity and the proportion of normal spermatozoa. Biol Reprod 2005; 72:822829 .

46. Januskauskas A, Rodriguez-Martinez H. Assessment of sperm viability by measurement of ATP, membrane integrity and motility in frozen thawed bull semen. Acta Vet Scand 1995; 36:571-574.

47. Stewart DL, Spooner RL, Bennett GH, Beatty RA, Hancock JL. Second experiment with heterospermic insemination in cattle. J Reprod Fertil 1974; 36:107-116.

48. Eggert-Kruse W, Schwarz H, Rohr G, Demirakca T, Tilgen W, RunneBaum B. Sperm morphology assessment using strict criteria and male fertility under in vivo conditions of conception. Hum Reprod 1996; 11: 139-146.

49. Yanagimachi R. Mammalian fertilization. In: Knobil E, Neill JD (eds.), The Physiology of Reproduction. New York: Raven Press; 1994:189-317.

50. Songsasen N, Leibo SP. Cryopreservation of mouse spermatozoa. II. Relationship between survival after cryopreservation and osmotic tolerance of spermatozoa from three strains of mice. Cryobiology 1997; 35:255-269.

51. Walters EM, Men H, Agca Y, Mullen SF, Critser ES, Critser JK. Osmotic tolerance of mouse spermatozoa from various genetic backgrounds: acrosome integrity, membrane integrity, and maintenance of motility. Cryobiology 2005; 50:193-205.

52. Willoughby CE, Mazur P, Peter AT, Critser JK. Osmotic tolerance limits and properties of murine spermatozoa. Biol Reprod 1996; 55:715-727.

53. Quintero-Moreno A, Miro J, Teresa Rigau A, Rodriguez-Gil JE. Identification of sperm subpopulations with specific motility characteristics in stallion ejaculates. Theriogenology 2003; 59:1973-1990.

54. Morton NE, Crow JF, Muller HJ. An estimate of the mutational damage in 
man from data on consanguineous marriages. Proc Natl Acad Sci U S A $1956 ; 42: 855-863$.

55. Saccheri IJ, Lloyd HD, Helyar SJ, Brakefield PM. Inbreeding uncovers fundamental differences in the genetic load affecting male and female fertility in a butterfly. Proc R Soc Lond B Biol Sci 2005; 272:39-46.

56. Reed DH, Nicholas AC, Stratton GE. Inbreeding levels and prey abundance interact to determine fecundity in natural populations of two species of wolf spider. Conserv Genet 2007; 8:1061-1071.

57. StatSoft. STATISTICA for Windows, Version 6.0. Tulsa, OK: StatSoft, Inc.; 2001.

58. Foote RH. Fertility estimation: a review of past experience and future prospects. Anim Reprod Sci 2003; 75:119-139.

59. Mooradian AD, Morley JE, Korenman SG. Biological actions of androgens. Endocr Rev 1987; 8:1-28.

60. Malo AF, Roldan ERS, Garde JJ, Soler AJ, Vicente J, Gortazar C, Gomendio M. What does testosterone do for red deer males? Proc R Soc Lond B Biol Sci 2009; 276:971-980.

61. Correa LM, Thomas A, Meyers SA. The macaque sperm actin cytoskeleton reorganizes in response to osmotic stress and contributes to morphological defects and decreased motility. Biol Reprod 2007; 77:942953.

62. Setty BS. Regulation of epididymal function and sperm maturationendocrine approach to fertility—control in male. Endokrinologie 1979; 74 : $100-117$.

63. Robaire B, Henderson NA. Actions of 5alpha-reductase inhibitors on the epididymis. Mol Cell Endocrinol 2006; 250:190-195.

64. Henderson NA, Cooke GM, Robaire B. Region-specific expression of androgen and growth factor pathway genes in the rat epididymis and the effects of dual 5alpha-reductase inhibition. J Endocrinol 2006; 190:779_ 791.

65. Christova Y, James PS, Cooper TG, Jones R. Lipid diffusion in the plasma membrane of mouse spermatozoa: changes during epididymal maturation, effects of $\mathrm{pH}$, osmotic pressure, and knockout of the c-ros gene. J Androl 2002; 23:384-392.

66. Henderson NA, Robaire B. Effects of PNU157706, a dual 5alpha- reductase inhibitor, on rat epididymal sperm maturation and fertility. Biol Reprod 2005; 72:436-443.

67. Plusquellec P, Bouissou MF. Behavioral characteristics of two dairy breeds of cows selected (Herens) or not (Brune des Alpes) for fighting and dominance ability. Appl Anim Behav Sci 2001; 72:1-21.

68. Hastings IM, Hill WG. The effect of testosterone in mice divergently selected on fat content or body weight. Genet Res 1997; 70:135-141.

69. Hooper AC, Brien TG, Lawlor PG. The effects of orchidectomy and the role of testosterone in determining the growth of male mice selected for increased body weight. Andrologia 1986; 18:509-515.

70. Olsson M, Wapstra E, Madsen T, Silverin B. Testosterone, ticks and travels: a test of the immunocompetence-handicap hypothesis in freeranging male sand lizards. Proc R Soc Lond B Biol Sci 2000; 267:2339_ 2343.

71. Straub RH, Tanko LB, Christiansen C, Larsen PJ, Jessop DS. Higher physical activity is associated with increased androgens, low interleukin 6 and less aortic calcification in peripheral obese elderly women. J Endocrinol 2008; 199:61-68.

72. Malo AF, Gomendio M, Garde JJ, Lang-Lenton B, Soler AJ, Roldan ERS. Sperm design and sperm function. Biol Lett 2006; 2:246-249.

73. Charlesworth B, Hughes KA. Age-specific inbreeding depression and components of genetic variance in relation to the evolution of senescence. Proc Natl Acad Sci U S A 1996; 93:6140-6145.

74. Hughes KA. The inbreeding decline and average dominance of genes affecting male life-history characters in Drosophila melanogaster. Genet Res 1995; 65:41-52.

75. Neubauer K, Jewgenow K, Blottner S, Wildt DE, Pukazhenthi BS. Quantity rather than quality in teratospermic males: a histomorphometric and flow cytometric evaluation of spermatogenesis in the domestic cat (Felis catus). Biol Reprod 2004; 71:1517-1524.

76. Joron M, Brakefield PM. Captivity masks inbreeding effects on male mating success in butterflies. Nature 2003; 424:191-194.

77. Meagher S, Penn DJ, Potts WK. Male-male competition magnifies inbreeding depression in wild house mice. Proc Natl Acad Sci U S A 2000; 97:3324-3329. 